\title{
VLC Based Indoor Tracking System with IOT Network for Smart Buildings
}

\author{
S.T. Santhanalakshmi, Bhavya Sree.S.K, Chittamuru Asrija, Deepthi.J
}

\begin{abstract}
Visible Light Communication (VLC)-based indoor localization approaches enjoy many advantages, such as utilizing ubiquitous lightening infrastructure, high location accuracy, and no interruption to RF-based devices ${ }^{6}$. Here our approach makes the location accuracy better than our existing methods. We used interior based receiver transmitter ${ }^{7}$ device to make our process in real time. In this concept we are going to monitor the persons details along with their location information. This information is sent to the server in periodic time. This makes the security of the building better.
\end{abstract}

Keywords:- Visible Light Communication, RF-based devices, Interior based transmitter

\section{INTRODUCTION}

Recently, the indoor positioning techniques have attracted considerable attention of the research community for providing location-based services. The high accuracy indoor positioning might open the doors to the exploration of novel applications and the creation of novel business opportunities in numerous scenarios such as augmented reality, tracking of patients in hospitals, personalized shopping, asset and personnel tracking. Precise indoor location estimation is indeed considered as one of the foundations in the realization of Internet of Things vision and $5 \mathrm{G}$ networks. The Global Positioning System (GPS) has been widely implemented in outdoor navigation systems. The location, velocity, and time information of any place on the Earth have been determined by an unobstructed line of sight from at least four GPS Satellites. Despite several advantages, Satellite signals, particularly in urban indoor areas under poor signal conditions may undergo multipath propagation. The signals can bounce off the structures, be weakened by the meteorological conditions, or get blocked by walls and high-rise buildings, resulting in an increase in the position errors of up to meters. These errors are considerable to allow accurately locating an occupant in a multi-storey building.

S.T. Santhanalakshmi, Bhavya Sree.S.K, Chittamuru Asrija, Deepthi.J: Department of Computer Science and Engineering, Panimalar Engineering College, Chennai.
Therefore, the GPS technique cannot meet the requirements of indoor positioning. There are several alternative techniques for indoor positioning such as radio frequency $(\mathrm{RF})$, infrared, ultrasonic, and image sensing. Among these techniques, it is well known that RF-based system has low cost and better coverage. However, their accuracy is still problematic because of the multipath fading. In addition, electromagnetic interference causes this technique to be restricted to certain RF-sensitive areas such as hospitals, aircrafts, and mines. Consequently, visible light communication (VLC) is noticed as an obvious technological candidate for improving indoor positioning capabilities. VLC is a reliable technology, which can offer high data rate, environment friendliness, and deliver high security. Specifically, VLC can deliver excellent accuracy of up to a few centimetres for indoor positioning. Moreover, VLC can be used as a ubiquitous communication because of the widespread prevalence of lighting devices.

\section{LITERATURE SURVEY}

J.C.Aguilar Herrera et al[1] proposes a pedestrian indoor positioning using smart phone multi-sensing, radio beacons, user positions probability map and indoor OSM floor plan representation. The system allows layering all kind of data to the existing map representation.

Jiabin Chen et al [2] proposes an indoor tracking algorithm based on particle filter and improved finger printing. In this system, the improved $\mathrm{KNN}$ algorithm is used to provide the position in a fast and precise way.

Shucai Wang et al [3] proposes a system with moving object detection and tracking in indoor environment with video recorded ahead experiments are realized and objects are tracked in real time.

Zenghua zhao et al[4] proposes navilight indoor localization and navigation under arbitrary lights. In this 
system, experiment shows that navilight can achieve submeter localisation accuracy to meet practical engineering requirements.

Sihua Shao et al [5] proposes a Pixelated VLCbackscattering for Self-charging indoor IOT devices. In general $\mathrm{n}$-fold throughput enhancement is realised by utilising $\mathrm{n}$ smaller VLC backscatters while incurring negligible energy using the same device as that of a single large backscatter.

\section{PROPOSED SYSTEM}

This paper aims to develop secure and reliable indoor tracking system. This is designed based on VLC and IOT. Our system has three modules,

\section{Transmitter module}

2.Receiver module

3.IOT module

Transmitter module is with the person it has IR transmitter to transmit the information about that person.

Receiver module is with the. interior wall mount this get the information from the transmitter and transmit to IoT server. IoT module receives the information from receiver module and stored as a $\log$.

\section{ARCHITECTURE DIAGRAM}

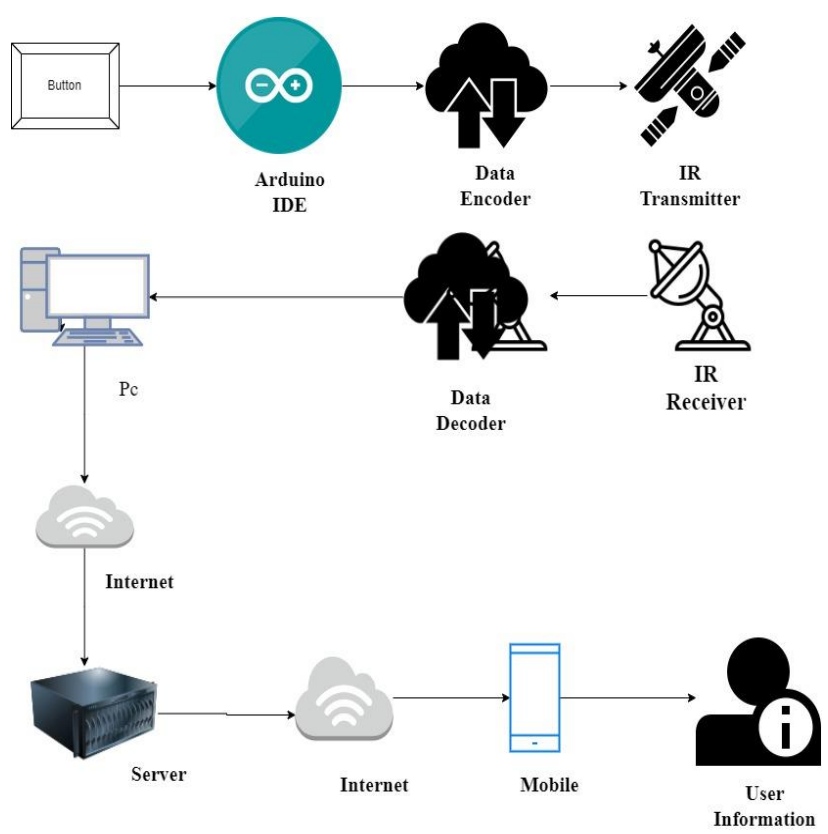

Fig: System structure of VLC based indoor tracking using IOT network

\section{Modules}

IR TRANSMITTER: An $I R$ transmitter contains an LED that emits infrared light. Thus the name. The receiver contains either a photodiode or a phototransistor (usually the latter). This component passes more or less current depending on the amount of $I R$ light falling on it. The LED is switched on and off in a coded sequence.

IR RECEIVER: An infrared receiver, or IR receiver, is hardware that sends information from an infrared remote control to another device by receiving and decoding signals. In general, the receiver outputs a code to uniquely identify the infrared signal that it receives

Pc: Stands for "Personal computer." PCs are what most of us use on a daily basis for work or personal use. most popular use for personal computers is for playing games. Businesses use personal computers for word processing, accounting, desktop publishing, and for running spreadsheet and database management applications. Image: Example of typical devices connected to a PC.

IoT: It is a system of interrelated computing devices, mechanical and digital machines, objects, animals or people that are provided with unique identifiers (UIDs) and the ability to transfer data over a network without requiring human-to-human or human-to-computer interaction.

LIFI: Here our LIFI transfer have a one TTL logic with LED light.This TTL logic unit controls the LED light based on the signal from Arduino transmitter . In receiver side solar panel receives the data and gives it to the Pc via Arduino UNO 


\section{FLOW ANALYSIS}

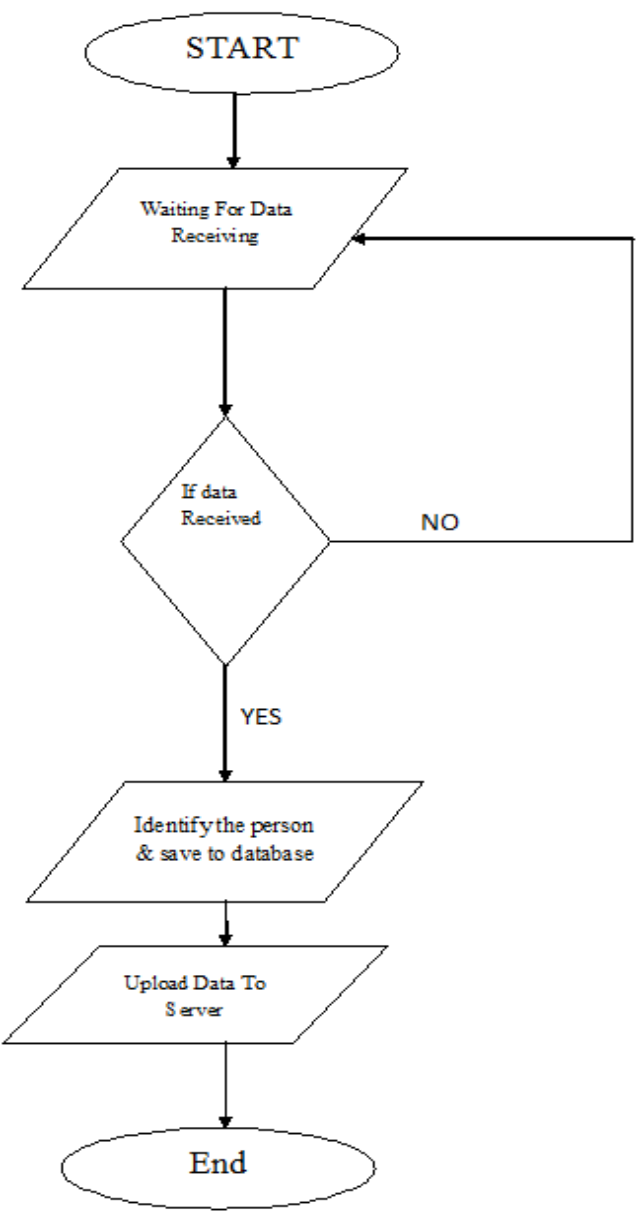

\section{ANALYSIS}

\section{Why we choose Arduino UNO?}

Cross-platform - The Arduino drivers and software runs on Mac, Windows, and Linux operating systems and they are completely open source. Most microcontroller systems are limited to Windows.

Inexpensive- Arduino boards are relatively inexpensive compared to other microcontroller platforms. The least expensive version of the Arduino module can be assembled by hand, and even the pre-assembled Arduino modules cost less than $\$ 50$ that comes with free authoring software.

Simple board - Many microcontroller boards are historically enormously complex with a lot of added-on parts like LCDs, buttons, LEDs, 7-segments, etc. showing everything it can do. Arduino has the bare minimum. Want more? Get a shield. There are hundreds of Arduino shields, from LCD to Wi-Fi, but it's up to the user to add that. Shields add extra functionality easily.

Simple programming environment - The Arduino programming environment is easy-to-use for beginners, yet flexible enough for advanced users to take advantage of as well. There are libraries to do simple things, like twiddle pins or debounce buttons and tons of objectwrapped libraries to do complex things, like writing to SD cards, LCD screens, parsing GPS. For teachers, it's conveniently based on the Processing programming environment, so students learning to program in that environment will be familiar with the look and feel of Arduino. Arduino is a simple system designed for creative people with little or "no prior knowledge of electronics. Above all, it has a very welcoming attitude towards beginners and tries not to scare them too much."

Open source and extensible software - The Arduino software is published as open source tools, available for extension by experienced programmers. The language can be expanded through $\mathrm{C}++$ libraries, and people wanting to understand the technical details can make the leap from Arduino to the AVR C programming language on which it's based. Similarly, you can add AVR-C code directly into your Arduino programs if you want to.

Sensor Data - The Arduino really took off because it has analog-to-digital input, in other words, you can take in sensor data like light, temperature, sound, or whatever using the low-cost sensors already on the market and get that into the Arduino easily. It also has ready-to-go SPI and I2C for digital sensors. This covers $99 \%$ of sensors on the market. You can't easily do this with other platforms.

\section{LABELS: ARDUINO, ARDUINO BASICS}

\section{Why we choose python?}

Python's simple, easy to learn syntax emphasizes readability and therefore reduces the cost of program maintenance.

Python supports modules and packages, which encourages program modularity and code reuse. 
The Python interpreter and the extensive standard library are available in source or binary form without charge for all major platforms, and can be freely distributed.

\section{CONCLUSION}

This paper presents an approach for visible light communication-based indoor positioning, visible light communication (VLC) has been gaining a good deal of attraction because of its ability to use the transmitterLED's for both communication and illumination. VLC can be used in an environment where wireless communication is not preffered such as hospitals and other chemical plants. This method proves we can identify each person or object in better accuracy.

\section{REFERENCES}

1. Armstrong, J.; Sekercioglu, Y.A.; Neild, A. Visible light positioning: A roadmap for international standardization. IEEE Commun. Mag. 2013, 51, 68-73. [CrossRef]

2. Rajagopal, S.; Roberts, R.D.; Lim, S. IEEE 802.15.7 visible light communication: Modulation schemes and dimming support. IEEE Commun. Mag. 2012, 50, 72-82. [CrossRef]

3. IEEE Draft Standard for Local and Metropolitan Area NetworksPart 15.7: Short-Range Optical Wireless Communications; IEEE P802.15.7/D3; Institute of Electrical and Electronics Engineers (IEEE): Englewood, CO, USA, 1 January 2018; pp. 1-412.

4. Keskin, M.F.; Sezer, A.D.; Gezici, S. Localization via Visible Light Systems. Proc. IEEE 2018, 106, 1063-1088.[CrossRef]

5. Indoor Positioning: Perfect Light, Precise Location. Available online: (accessed on 3 February 2019).

6. Qiu, K.; Zhang, F.; Liu, M. Let the Light Guide Us: VLC-Based Localization. IEEE Robot. Autom. Mag. 2016, 23, 174-183. [CrossRef] 7. Murai, R.; Sakai, T.; Kawano, H.; Matsukawa, Y.; Kitano, Y.; Honda, Y.; Campbell, K.C. A novel visible light communication system for enhanced control of autonomous delivery robots in a hospital. In Proceedings of the 2012 IEEE/SICE International Symposium on System Integration (SII), Fukuoka, Japan, 16-18 December 2012; pp. 510-516. [CrossRef]

8. Zhuang, Y.; Hua, L.; Qi, L.; Yang, J.; Cao, P.; Cao, Y.; Wu, Y.; Thompson, J.; Haas, H. A Survey of Positioning Systems Using Visible LED Lights. IEEE Commun. Surv. Tutor 2018, 20, 1963-1988. [CrossRef]

9. Do, T.-H.; Yoo, M. An in-Depth Survey of Visible Light Communication Based Positioning Systems. Sensors 2016, 16, 678. [CrossRef]

10. Yang, C.; Shao, H.WiFi-based indoor positioning. IEEE Commun. Mag. 2015, 53, 150-157. [CrossRef] 